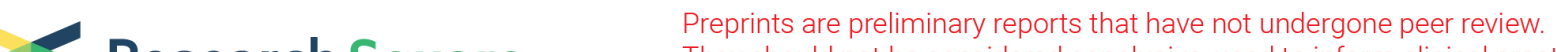 $\begin{array}{ll}\text { Research Square } & \begin{array}{l}\text { They should not be considered conclusive, used to inform clinical practice, } \\ \text { or referenced by the media as validated information. }\end{array}\end{array}$
}

\section{Differences in the Microsurface and Microvascular Features between Early Gastric Cancer of Corpus and Antrum under Narrow Band Imaging Magnifying Endoscopy}

\section{Qian Zhuang}

Shanghai General Hospital of Nanjing Medical University

Jing Wang

Shanghai Jiaotong University First People's Hospital Department of Pathology

Shengzheng Luo

Shanghai General Hospital, Shanghai Jiao Tong University School of Medicine

\section{Xiaowan Wu}

Shanghai General Hospital of Nanjing Medical University

Jinnian Cheng

Shanghai General Hospital of Nanjing Medical University

\section{Yueqin Qian}

Shanghai General Hospital, Shanghai Jiao Tong University School of Medicine

\section{Xiaoyuan Gong}

Shanghai General Hospital, Shanghai Jiao Tong University School of Medicine

Hui Zhou

Shanghai General Hospital, Shanghai Jiao Tong University School of Medicine

Xinjian Wan ( $\sim$ mdwanxinjian@126.com )

Shanghai General Hospital, Shanghai Jiao Tong University School of Medicine

\section{Research article}

Keywords: Magnifying endoscopy; Narrow band imaging; Early gastric cancer; Antrum; Corpus

Posted Date: December 13th, 2019

DOI: https://doi.org/10.21203/rs.2.13670/v2

License: () (1) This work is licensed under a Creative Commons Attribution 4.0 International License.

Read Full License 


\section{Abstract}

Background Microsurface structure (MS) and microvascular features (MV) of early gastric cancer (EGC) are associated with the histologic type, invasion depth and macroscopic shape of lesions. However, no studies have reported the differences in the MS and MV features between EGCs of antrum and corpus under magnifying endoscopy with narrow band imaging (ME-NBI).Methods This was a retrospective study including 116 patients with EGC. The characteristics of MS and MV pattern of EGC were evaluated in 2 group, antrum type and corpus type.Results According to the Sakaki's classification, the MS of EGCs presented as IV, V1 or VI typein in sequence, one level higher than the previous type in the degree of atypia. Tumor location and differentiated degree were identified as independent predictors for MS pattern. For the probability of at least one level higher, in the MS pattern, the corporal EGCs was 5.84 times as high as that of antral EGCs ( $\chi 2=9.42, P=0.002,95 \% \mathrm{Cl} 1.89-18.05)$, and undifferentiated-type lesions was 8.82 times as high as that of the differentiated-type lesions ( $\chi 2=11.67, \mathrm{P}=0.001,95 \% \mathrm{Cl} 2.53-30.76$ ). No difference in the MV pattern was observed between antral EGCs and corporal EGCs $(P=0.7)$, while the microvascular pattern and microsurface pattern (VS pattern, FNP, ILL-1, ILL-2 and CSP) were significantly different between those 2 groups $(P=0.001)$. Conclusions There are significant differences in the MS and VS pattern of EGC between antrum and corpus types. The antral EGC is less obvious than the corporal $E G C$, therefore, it is more likely to be missed in clinical gastroscopy.

\section{Background}

Magnifying endoscopy with narrow band imaging (ME-NBI) is able to observe the features of microsurface and microvessels in the gastric mucosa. Studies have confirmed the value of ME-NBI in the diagnosis of early gastric cancer (EGC) [1-4]. Prior studies have demonstrated that ME-NBI can achieve endoscopic pathology of the upper gastrointestinal tract, in which an accurate endoscopic diagnosis comparable to the histopathological diagnosis can be made without biopsy [1, 5-7]. That is to say, ME$\mathrm{NBI}$ can provide very clear endoscopic images in which critical histopathological features (such as structural atypia) can be identified [8]. It has also been proven to be able to predict the histological characteristics of gastric cancer lesions, such as the degree of differentiation [9], the depth of infiltration $[10,11]$, etc. ME-NBI can also assist to determine the tumor boundaries and avoid positive postoperative surgical margins [12]. Therefore, it is an effective method for preoperative evaluation of tumors.

Microsurface structure (MS) and microvascular features (MV) of EGC are associated with the macroscopic shape, histologic type and invasion depth of lesions [13]. It is well known that there are significant differences in MV and MS between the mucosa of normal antrum and corpus under ME-NBI [4, 14]. Normal corporal mucosa displays a regular honeycomb-like subepithelial capillary network (SECN) and regular oval crypt opening with circular marginal crypt epithelium (MCE) [14]. Normal antral mucosa appears as a regular coil-shaped SECN pattern and regular polygonal or curved MCE pattern [14]. However, no studies have reported the difference between EGCs of the corpus and antrum under ME-NBI. The MV and MS features of EGC summarized in previous studies also cannot be used to distinguish 
between the different regions of antrum and corpus[13, 15-18]. We hypothesize that there may be different MV and MS patterns between EGCs of the corpus and antrum under ME-NBI.

\section{Methods}

\section{Study design and patient selection}

This retrospective study was carried out at a single tertiary referral center in Shanghai China in conformity to the declaration of Helsinki, and was approved by the institutional review board of Shanghai General Hospital of China.

The patients meeting the following inclusion and exclusion criteria were enrolled into the present study from Shanghai General Hospital between September 2014 and March 2019. ME-NBI was conducted before treatments and the images were stored in a digital filing system. All patients underwent endoscopic submucosal resection or surgical treatment and were histologically diagnosed with EGC. Lesions with inappropriate NBI images due to bleeding, ulcer covered by corpus albicans, or excessive mucosal congestion were excluded from the study. Finally, a total of 116 EGCs from 116 patients were included in the present study.

\section{ME-NBI}

ME-NBI was performed with a magnifying endoscope (GIFH260Z, Olympus Co., Ltd, Japan) (GIFH260Z, Olympus, Tokyo, Japan) equipped with a video processor (EVIS LUCERA Olympus CV-290, Olympus Co. Ltd), and a light source (EVIS LUCERA Olympus CLV-290SL, Olympus co.). The structure enhancement of the endoscopic video processor was set at B-mode level 8 for ME-NBI. The color mode was fixed at level 1. To obtain stable endoscopic images at maximal magnification, a black, soft hood attachment (MAJ1990, Olympus co.) was mounted at the tip of the endoscope before examination.

The MS and MV of EGC areas were retrospectively evaluated using ME-NBI images captured at the maximal magnification by an experienced endoscopist who was blind to the histological findings.

Studies have reported that epithelial structures can be observed on ME-NBI $[2,6,9]$. In the present study, structural atypia was used to describe the degree of microsurface structure disorder. According to the Sakaki types [19], the magnifying images of EGC areas were classified into three types[20]: IV, $\mathrm{V}_{1}$ and $\mathrm{VI}$ based on MS patterns. Type IV is ovoid or patchy and type $\mathrm{V}_{1}$ is finger or villous structure. The microsurface structure of type VI is often disordered, destroyed or even absent. As the increase in the classification level, the microsurface structure becomes more disordered and is more prone to be destroyed and absent or in other words the grade of atypia of microsurface structure gets higher (Fig. 1). The MV patterns were classified as loop, fine network, or corkscrew according to the classification previously reported [16] (Fig. 2). 
According to the classification proposed by Yokoyama et al [13], NBI magnifying images were also classified into microvascular pattern and microsurface pattern (VS patterns): FNP, ILL-1, ILL-2 and CSP, which were simultaneously based on the abnormal VS patterns (Fig. 3). The predominant pattern was used for classification in case of lesions with combined MS or MV patterns.

\section{Histological assessment}

The removed tumors were fixed in $10 \%$ buffered formalin for $24 \mathrm{~h}$ and then longitudinally cut into $2-3 \mathrm{~mm}$ slices. These sliced were embedded in paraffin and stained with hematoxylin and eosin for pathologic examination. In the present study, the revised Vienna [21] categories 4 and 5 were used to define EGC. The depth of invasion and degree of differentiation were assessed microscopically. The histologic diagnosis of EGC was according to WHO classification [22]. The WHO classification is commonly divided into 2 major categories in clinical study: differentiated and undifferentiated type (Nakamura's classification) [23, 24]. The former includes well-differentiated adenocarcinoma, moderately-differentiated adenocarcinoma, and papillary adenocarcinoma, and the latter includes poorly-differentiated adenocarcinoma, signet-ring cell carcinoma, and mucinous adenocarcinoma. In this study, we utilized this classification method to divide EGC into two types: differentiated and undifferentiated type.

\section{Statistical analysis}

Because the Sakaki types of MS patterns were ordered categories, Wilcoxon rank-sum test was used in univariate analysis, and ordinal ploytomous logistic regression was used in multivariate analysis, so as to compare the degree of atypia of MS between different groups. However, unlike MS patterns, the types of MV patterns and VS patterns were unordered categories and their distribution between different groups was analyzed using the Fisher exact test. Statistical analyses were performed with IBM SPSS Statistics for Windows (Version 24.0) and the free software R (www.r-project.org). A value of $P<0.05$ was considered statistically significant.

\section{Results}

\section{Participants}

In total, 116 EGC lesions were examined in the present study. Thirty-nine lesions located in the corpus and 77 in the antrum. Fifty-six lesions were treated via ESD and 60 via surgery. The results of histologic assessment based on histologic type and invasion depth were as follows: 70 lesions were differentiatedtype EGCs (D-EGCs) and 46 undifferentiated-type EGCs (U-EGCs); 95 lesions were mucosal cancers and 21 submucosal cancers (Table 1 ).

\section{Table 1 Clinicopathologic Characteristics of EGCs at Baseline}




\begin{tabular}{|c|c|}
\hline $\begin{array}{l}\text { Characteristics } \\
\text { No. of patients } \\
\text { Age, year }\end{array}$ & $\begin{array}{c}\text { Value } \\
116 \\
64(33-83) \\
\end{array}$ \\
\hline $\begin{array}{cc}\text { Sex } & \text { Male } \\
& \text { Female } \\
\end{array}$ & $\begin{array}{l}75(64.7) \\
41(35.3) \\
\end{array}$ \\
\hline $\begin{array}{r}\text { Tumor location } \\
\text { Antrum } \\
\text { Corpus }\end{array}$ & $\begin{array}{l}77(66.4) \\
39(33.6)\end{array}$ \\
\hline $\begin{array}{l}\text { Macroscopic shape } \\
\text { elevated-type } \\
\text { flat- and depressed-type }\end{array}$ & $\begin{array}{l}49(42.2) \\
67(57.8)\end{array}$ \\
\hline $\begin{array}{l}\text { Histologic type } \\
\text { Differentiated-type } \\
\text { Undifferentiated-type }\end{array}$ & $\begin{array}{l}70(60.3) \\
46(39.7) \\
\end{array}$ \\
\hline $\begin{array}{c}\text { Depth of invasion } \\
\text { Mucosa } \\
\text { Submucosa } \\
\end{array}$ & $\begin{array}{l}95(81.9) \\
21(18.1) \\
\end{array}$ \\
\hline $\begin{array}{c}\text { Treatment } \\
\text { Endoscopic resection } \\
\text { Surgery } \\
\end{array}$ & $\begin{array}{l}56(48.3) \\
60(51.7) \\
\end{array}$ \\
\hline
\end{tabular}

Data are presented as number (\%) or mean (range).

According to the lesion shape reported by Yokoyama et al [13], the lesions were classified into 2 groups according to their morphology: elevated-type EGC (type 0-I, type Ila, and type Ila+Ilc; predominantly Ila); and flat- and depressed-type EGC that consisted of type Ilb and type Ilc. As a result, 49 lesions were classified as elevated-type EGC and 67 lesions as flat- and depressed-type EGC.

\section{MS patterns and tumor location of EGCs}

As shown in Table 2, tumor location $(P<0.001)$, histologic type $(P<0.001)$, invasion depth $(P=0.002)$ and macroscopic shape $(P=0.03)$ were significantly associated with the types of MS patterns (the degree of structural atypia) in univariate analysis.

Table 2 Associations between clinical characteristics and types of MS pattern

\begin{tabular}{|c|c|c|c|c|}
\hline & $I V$ & $\overline{V_{1}}$ & $\overline{V I}$ & $P$-value \\
\hline Tumor location & & & & $<0.001$ \\
\hline Corpus & $3(7.7)$ & $2(5.1)$ & $34(87.2)$ & \\
\hline Antrum & $30(39.0)$ & $6(7.8)$ & $41(53.2)$ & \\
\hline Histologic type & & & & $<0.001$ \\
\hline Undifferentiated-type & $3(6.5)$ & $1(2.2)$ & $42(91.3)$ & \\
\hline Differentiated-typ & $30(42.9)$ & $7(10.0)$ & $33(47.1)$ & \\
\hline Depth of invasion & & & & 0.002 \\
\hline Mucosa & $32(33.7)$ & $8(8.4)$ & $55(57$ & \\
\hline Submucosa & & $0(0.0)$ & & \\
\hline Macroscopic shape & & & & 0.03 \\
\hline Elevated-type & $18(36.7)$ & $5(10.2)$ & $26(5$ & \\
\hline Flat- and depressed-type & $15(2$ & $.5)$ & 1967 & \\
\hline
\end{tabular}


In Table 3, multivariate analysis showed tumor location and histologic type were independent predictors for MS structure. For the classification categories of MS patterns in EGCs were at least one grade higher, the corpus was 5.84 times of the possibility as the antrum $\left(c^{2}=9.42, \mathrm{P}=0.002,95 \% \mathrm{Cl} 1.89-18.05\right)$, and undifferentiated-type EGCs was 8.82 times of the possibility as the differentiated-type EGCs $\left(c^{2}=11.67\right.$, $\mathrm{P}=0.001,95 \% \mathrm{Cl} 2.53-30.76$ ). In simple terms, if comparing the possibility that the MS patterns of EGCs belong to the higher classification categories, corpus was greater than antrum (Fig. 4-6) and U-EGCs was greater than D-EGCs.

Table 3 Ordinal ploytomous logistic regression of different types of MS patterns

\begin{tabular}{lccllccc}
\hline & $\beta$ & $S E$ & Wald $c^{2}$ & $P$-value & OR & OR & 95\%CI \\
\hline Tumor location & 1.77 & 0.58 & 9.42 & 0.002 & 5.84 & 1.89 & 18.05 \\
Histologic type & 2.18 & 0.64 & 11.67 & 0.001 & 8.82 & 2.53 & 30.76 \\
Depth of invasion & 1.27 & 1.14 & 1.24 & 0.27 & 3.56 & 0.38 & 33.27 \\
Macroscopic shape & 0.28 & 0.46 & 0.36 & 0.55 & 1.32 & 0.53 & 3.28 \\
\hline
\end{tabular}

SE, Standard Error; OR, Odds ratio; CI, confidence interval.

\section{MV patterns, VS patterns and tumor location of EGCs}

It was reported that MV patterns and VS patterns were associated with the histologic type, invasion depth and macroscopic shape of lesions [13], which were confounding factors when the location factor was assessed. Before Case Control Matching, the distribution of invasion depth was significantly associated with the tumor location of EGCs $\left(c^{2}=8.0, P=0.005\right)$ (Table 4-1). In order to maintain a balance between antrum group and corpus group, case control matching 1:1 according to histologic type (differentiatedtype or undifferentiated type), invasion depth (mucosa or submucosa) and macroscopic shape (elevatedtype or flat- and depressed-type) was used, and thus there were 33 pair of exact matches finally. After adjustment for confounding factors, there was no significant difference in histologic type, invasion depth and macroscopic shape between those 2 groups (Table 4-2).

Table 4-1 The distribution of histologic type, invasion depth and macroscopic shape between antrum and corpus group before case control matching 1:1 


\begin{tabular}{llllc}
\hline & Corpus & Antrum & $c^{2}$ & $P$-value \\
\hline $\begin{array}{l}\text { Histologic type } \\
\text { Undifferentiated-type }\end{array}$ & $\begin{array}{l}19(48.7) \\
20(51.3)\end{array}$ & $\begin{array}{l}27(35.1) \\
50(64.9)\end{array}$ & \multirow{2}{*}{1.0} & 0.2 \\
$\quad$ Differentiated-type & $\begin{array}{l}26(66.7) \\
13(33.3)\end{array}$ & $\begin{array}{l}69(89.6) \\
8(10.4)\end{array}$ & \multirow{2}{*}{8.0} & 0.005 \\
\hline $\begin{array}{l}\text { Invasion depth } \\
\text { Mucosa }\end{array}$ & $\begin{array}{l}14(35.9) \\
\text { Submucosa }\end{array}$ & $\begin{array}{l}35(45.5) \\
42(54.5)\end{array}$ & 0.6 & 0.4 \\
\hline $\begin{array}{l}\text { Macroscopic shape } \\
\text { Elevated-type }\end{array}$ & & & & \\
$\quad$ Flat- and depressed-type & $25(64.1)$ & &
\end{tabular}

Table 4-2 The distribution of histologic type, invasion depth and macroscopic shape between antrum and corpus group after case control matching 1:1

\begin{tabular}{lccc}
\hline & Corpus & Antrum & $P$-value \\
\hline Histologic type & & & \\
Undifferentiated-type & 15 & 15 & 1 \\
Differentiated-type & 18 & 18 & \\
\hline Depth of invasion & 26 & 26 & 1 \\
Mucosa & 7 & 7 & \\
Submucosa & 13 & 13 & 1 \\
\hline Macroscopic shape & 20 & 20 & \\
Elevated-type & \\
Flat- and depressed-type & 20 &
\end{tabular}

\section{MS patterns and tumor location of EGCS}

Although the number of cases got smaller after case control matching 1:1, MS patterns were still significantly different between corpus group and antrum group $(P=0.009)$, which was consistent with the above result of ordinal ploytomous logistic regression (Table 5).

Table 5 Association between MS patterns and tumor location (n; \%).

\begin{tabular}{lllll}
\hline & $I V$ & $V_{1}$ & $V I$ & $P$-value \\
\hline Corpus & $3(9.1)$ & $2(6.1)$ & $28(84.8)$ & 0.009 \\
Antrum & $13(39.4)$ & $2(6.1)$ & $18(54.5)$ & \\
\hline
\end{tabular}

MV patterns and tumor location of EGCs

As shown in Table 6, there was no difference in the MV patterns of EGCs between antrum group and corpus group $(\mathrm{P}=0.7)$.

Table 6 The MV patterns and tumor location (n; \%) 


\begin{tabular}{lccrr}
\hline & fine network & loop & corkscrew & P-value \\
\hline Corpus & $7(21.2)$ & $13(39.4)$ & $13(39.4)$ & 0.7 \\
Antrum & $4(12.1)$ & $15(45.5)$ & $14(42.4)$ & \\
\hline
\end{tabular}

VS patterns and tumor location of EGCS

As shown in Table 7, the classification categories were significantly different between corpus group and antrum group $(P=0.001)$. The proportion of FNP in the corpus was higher than in antrum group, while the proportion of ILL-1 in antrum group was much higher than corpus group. There was no significant difference in the distribution of ILL-2 and CSP between those two groups.

Table 7 Association between MS and MV patterns and tumor location (n; \%).

\begin{tabular}{llcccr}
\hline & $F N P$ & $I L L-1$ & ILL-2 & CSP & P-value \\
\hline Corpus & $7(21.2)$ & $45(15.2)$ & $11(33.3)$ & $10(30.3)$ & 0.001 \\
Antrum & $0(0)$ & $17(51.5)$ & $7(21.2)$ & $9(27.3)$ & \\
\hline
\end{tabular}

\section{Discussion}

In the present study, results showed that the MS patterns of EGC on me-nbi were significantly different in the gastric antrum and the corpus. Compared with the antral lesions, the grade of atypia in MS patterns was higher in corpus group. Based on the VS classification system, antral lesions were more likely to be ILL-1 type, while the corporal lesions were more inclined to be FNP type in those 2 groups of EGC.

In this study, the gastric pit pattern was evaluated according to the system proposed by Sakaki et al [19]. Although there are some limitations in this system, currently there is no other internationally recognized classification system for the assessment of gastric pits on upper digestive tract magnifying endoscopy. Therefore, this classification is still widely used in clinical practice [25]. According to the classification, the types of gastric pit pattern of EGC or precancerous gastric lesions mainly presented as IV, $\mathrm{V}_{1}$ or VI type [20]. In the present study, we found that the grade of atypia in MS patterns in the corpus type was more likely to be higher than in the antrum. In other words, the disordered or missing MS patterns in the corpus group were more obvious than the antrum group (Fig. 4-6).

That's an interesting finding. It is known that structural atypia and cellular atypia are key histopathological features for cancer diagnoses. ME-NBI can produce optical images reflecting structural atypia, which can achieve "optical biopsy" [8]. The structural atypia of gastric gland (or MS) of EGC may present as disordered or absent under ME-NBI. In that way, what's the reason that the degree of MS atypia in the corpus group was significantly higher than that in the antrum group?

We speculate that this phenomenon could be explained by the structure of gastric wall mucosa, the occurrence of tumor epithelium, and the characteristics of tissue atypia. Histologically, the gastric pit comprises approximately $50 \%$ of antral mucosal thickness, but only $25 \%$ of corporal mucosal thickness. 
There is evidence showing that gastric stem cells usually reside in the base of gastric unit and gastric cancer stem cells (GCSCs) play an important role in the occurrence and development of gastric cancer [26]. Thus, we assume that the proliferation of GCSCs may cause gradual glandular morphological changes which arise from the bottom. The superficial glandular structure in the corpus is more susceptible to these changes because the crypts in the corporal mucosa are shallower. When the gastric mucosa becomes cancerous, corporal microsurface is prone to be disorganized, which can be easily observed on ME-NBI.

Previous researches have also proposed that the degree of differentiation of tumor tissue affects the appearance of the microsurface [9, 27]. Kanesaka et al [9] reported that the absence of MS patterns was the characteristic of U-EGCs because cancer cells in U-EGC usually failed to form glands and the original epithelial structure was often destroyed, while cancer cells in D-EGCs usually had epithelial glandular structures, which showed subtle structural atypia. Our study also suggested the structural atypia of MS patterns in U-EGCs was significantly different from that in D-EGCs, which was consistent with the pathological features of those 2 types of cancers.

However, the relationship between histological type factors and magnifying images may also be influenced by other common factors, such as depth of invasion [10], macroscopic morphology [13], and tumor location. Similarly, the relationship between tumor location and microstructure of EGC under menbi was also affected by other confounding factors, including depth of invasion and macroscopic morphology, in addition to histological type. In order to comprehensively analyzed whether those four factors were correlated with MS patterns, a common statistical method for multivariate analysis was employed to assess the whole cases in this study. The analysis confirmed that only tumor location and histologic type were independent predictors for MS patterns (Table 3).

As a result, in the EGCs, compared with the corpus, the atypia of MS pattern EGCs in the antrum were more subtle and thus their diagnosis might be more likely to be missed clinically. In daily endoscopic examination, there are usually multiple lesions coexisting in the gastric antrum, while EGC may only show slight changes in microstructure and microvascular patterns [28]. EGCs in the gastric antrum area are easily missed or difficult to distinguish from non-cancerous lesions due to the above reasons. Therefore, when equivocal lesions are found in the antrum, additional biopsies may be essential for inexperienced endoscopist.

Because the MV patterns and VS patterns were associated with the histologic type, invasion depth and macroscopic shape of lesions $[9,13]$, case control matching was employed to adjust these confounding factors before further analysis of tumor location. As a result, according to the MV classification used in this study, there was no significant difference in MV patterns between EGCs in the antrum and corpus. The microvessels in the superficial cancerous epithelium are deformed as a result of compression by the structurally atypical cancerous glands [29]. Thus, the secondary changes may not be different between the antrum type and the corpus type. 
Yokoyama et al [13] reported that the distribution of VS patterns was significantly different between DEGCs and U-EGCs. In this study, the classification categories between D-EGCs and U-EGCs were consistent with those reported by Yokoyama et al (Additional file 1: Table S1). As a result, the distribution of VS patterns was also significantly different between the corpus type and the antrum type of EGC. As we know, normal corporal mucosa appears as a regular honeycomb-like SECN pattern and regular oval crypt opening with circular MCE pattern [14]. Normal antral mucosa displays a regular coil-shaped SECN pattern and regular polygonal or curved MCE pattern [14]. FNP and ILL-1 are predominantly found in differentiated-type adenocarcinomas [13], in which there are still epithelial glandular structures with subtle structural atypia. Morphologically, FNP is similar to the normal corpus MS pattern and ILL-1 to the normal antral MS pattern. Correspondingly, in EGC lesions, FNP is mainly observed in the corpus type, while ILL-1 is more common in the antrum type.

However, the incidence of ILL-2 and CSP in the gastric antrum and gastric corpus is comparable. Based on previous work [13] and our findings (Additional file 1: Table S1), ILL-2 and CSP are mainly found in undifferentiated adenocarcinoma. We hypothesize that, since their glandular and epithelial structures are often destroyed in U-EGCs, the pattern of microstructure may not differ greatly between the antrum type and the corpus type. In clinical practice, these findings are helpful for the better understanding of the likelihood of these four VS categories in different sites and degrees of differentiation of EGCs.

There are also some limitations in this study. First, this was a retrospective study from a single center, and the sample size was relatively small. More multicenter, prospective studies are needed to confirm our findings. Second, no significant difference was observed between the MV patterns of EGCs in the antrum and corpus in the present study, according to the current MV classification. Whether this is related to the method used for classification of MV patterns is unclear.

\section{Conclusions}

In conclusion, there are some differences in the manifestations of EGC in the gastric antrum and corpus on ME-NBI. The atypia in the patterns of MS and VS in the antral lesions is less evident than in the corporal lesions. This may be helpful for the diagnosis of EGC on gastroscopy in clinical practice.

\section{Abbreviations}

MS: Microsurface structure; MV: microvascular features; EGC: Early gastric cancer; ME-NBI: magnifying endoscopy with narrow band imaging; VS pattern: microvascular pattern and microsurface pattern; SECN: subepithelial capillary network; MCE: marginal crypt epithelium; D-EGCs: differentiated-type EGCs; UEGCs: undifferentiated-type EGCs; GCSGs: gastric cancer stem cells

\section{Declarations}

Acknowledgements 
Not applicable.

\section{Funding}

This work has been supported by the Shanghai municipal commission of science and technology of China (The grant number 18411952900).

\section{Availability of data and materials}

The datasets used and/or analyzed during the current study are available from the corresponding author on reasonable request.

\section{Authors' contributions}

$\mathrm{XW}, \mathrm{HZ}$ supervised the entire study and participated in study design and coordination. QZ, JW, SL, XW, JC, $Y Q$, and $X G$ performed endoscopic operation, pathological assessment and Case evaluation. QZ performed statistical analyses and drafted the manuscript. All authors read and approved the final manuscript.

\section{Ethics approval and consent to participate}

This retrospective study was approved by the institutional review board of Shanghai General Hospital of China. The waiver of individual patient informed consent was obtained due to minimal risk nature of this study.

\section{Consent for publication}

Not applicable.

\section{Competing interests}

The authors declare that they have no competing interests

\section{References}

1. Kaise M, Kato M, Urashima M, Arai Y, Kaneyama H, Kanzazawa Y, Yonezawa J, Yoshida Y, Yoshimura $\mathrm{N}$, Yamasaki T et al: Magnifying endoscopy combined with narrow-band imaging for differential diagnosis of superficial depressed gastric lesions. Endoscopy 2009, 41(4):310-315.

2. Kanesaka T, Sekikawa A, Tsumura T, Maruo T, Osaki Y, Wakasa T, Shintaku M, Yao K: Dense-type crypt opening seen on magnifying endoscopy with narrow-band imaging is a feature of gastric adenoma. Dig Endosc 2014, 26(1):57-62.

3. Yamada S, Doyama H, Yao K, Uedo N, Ezoe Y, Oda I, Kaneko K, Kawahara Y, Yokoi C, Sugiura Y et al: An efficient diagnostic strategy for small, depressed early gastric cancer with magnifying narrow- 
band imaging: a post-hoc analysis of a prospective randomized controlled trial. Gastrointest Endosc 2014, 79(1):55-63.

4. Muto $M$, Yao K, Kaise M, Kato M, Uedo N, Yagi K, Tajiri H: Magnifying endoscopy simple diagnostic algorithm for early gastric cancer (MESDA-G). Dig Endosc 2016, 28(4):379-393.

5. Nakayoshi T, Tajiri H, Matsuda K, Kaise M, Ikegami M, Sasaki H: Magnifying endoscopy combined with narrow band imaging system for early gastric cancer: correlation of vascular pattern with histopathology (including video). Endoscopy 2004, 36(12):1080-1084.

6. Yao K, Anagnostopoulos GK, Ragunath K: Magnifying endoscopy for diagnosing and delineating early gastric cancer. Endoscopy 2009, 41(5):462-467.

7. Yagi K, Nozawa Y, Endou S, Nakamura A: Diagnosis of Early Gastric Cancer by Magnifying Endoscopy with NBI from Viewpoint of Histological Imaging: Mucosal Patterning in terms of White Zone Visibility and Its Relationship to Histology. Diagnostic and therapeutic endoscopy 2012, 2012:954809.

8. Kaise M: Advanced endoscopic imaging for early gastric cancer. Best practice \& research Clinical gastroenterology 2015, 29(4):575-587.

9. Kanesaka T, Sekikawa A, Tsumura T, Maruo T, Osaki Y, Wakasa T, Shintaku M, Yao K: Absent microsurface pattern is characteristic of early gastric cancer of undifferentiated type: magnifying endoscopy with narrow-band imaging. Gastrointest Endosc 2014, 80(6):1194-1198 e1191.

10. Kobara H, Mori H, Fujihara S, Kobayashi M, Nishiyama N, Nomura T, Kato K, Ishihara S, Morito T, Mizobuchi $\mathrm{K}$ et al: Prediction of invasion depth for submucosal differentiated gastric cancer by magnifying endoscopy with narrow-band imaging. Oncol Rep 2012, 28(3):841-847.

11. Kikuchi D, lizuka T, Hoteya S, Yamada A, Furuhata T, Yamashita S, Domon K, Nakamura M, Matsui A, Mitani $T$ et al: Usefulness of magnifying endoscopy with narrow-band imaging for determining tumor invasion depth in early gastric cancer. Gastroenterol Res Pract 2013, 2013:217695.

12. Horiuchi Y, Fujisaki J, Yamamoto N, Shimizu T, Omae M, Ishiyama A, Yoshio T, Hirasawa T, Yamamoto $Y$, Tsuchida $T$ et al: Accuracy of diagnostic demarcation of undifferentiated-type early gastric cancer for magnifying endoscopy with narrow-band imaging: surgical cases. Surg Endosc 2017, 31(4):1906-1913.

13. Yokoyama A, Inoue H, Minami H, Wada Y, Sato Y, Satodate H, Hamatani S, Kudo SE: Novel narrowband imaging magnifying endoscopic classification for early gastric cancer. Dig Liver Dis 2010, 42(10):704-708.

14. Yao K: Clinical Application of Magnifying Endoscopy with Narrow-Band Imaging in the Stomach. Clin Endosc 2015, 48(6):481-490.

15. Okada K, Fujisaki J, Kasuga A, Omae M, Hirasawa T, Ishiyama A, Inamori M, Chino A, Yamamoto Y, Tsuchida $\mathrm{T}$ et al: Diagnosis of undifferentiated type early gastric cancers by magnification endoscopy with narrow-band imaging. J Gastroenterol Hepatol 2011, 26(8):1262-1269.

16. Ok KS, Kim GH, Park do Y, Lee HJ, Jeon HK, Baek DH, Lee BE, Song GA: Magnifying Endoscopy with Narrow Band Imaging of Early Gastric Cancer: Correlation with Histopathology and Mucin 
Phenotype. Gut Liver 2016, 10(4):532-541.

17. Hamada K, Itoh T, Kawaura K, Azukisawa S, Kobayashi R, Okamura H, Kitakata H, Urashima S, Nojima T, Fujino MA: Findings of the margin around lesions by magnifying endoscopy with narrowband imaging in early gastric carcinoma and intestinal metaplasia. J Dig Dis 2016, 17(6):377-382.

18. Matsuo K, Takedatsu H, Mukasa M, Sumie H, Yoshida H, Watanabe Y, Akiba J, Nakahara K, Tsuruta $\mathrm{O}$, Torimura T: Diagnosis of early gastric cancer using narrow band imaging and acetic acid. World $\mathrm{J}$ Gastroenterol 2015, 21(4):1268-1274.

19. Sakaki N, lida Y, Okazaki Y, Kawamura S, Takemoto T: Magnifying endoscopic observation of the gastric mucosa, particularly in patients with atrophic gastritis. Endoscopy 1978, 10(4):269-274.

20. Zhang J, Guo SB, Duan ZJ: Application of magnifying narrow-band imaging endoscopy for diagnosis of early gastric cancer and precancerous lesion. BMC gastroenterology 2011, 11:135.

21. Schlemper RJ, Riddell RH, Kato Y, Borchard F, Cooper HS, Dawsey SM, Dixon MF, Fenoglio-Preiser CM, Flejou JF, Geboes K et al: The Vienna classification of gastrointestinal epithelial neoplasia. Gut 2000, 47(2):251-255.

22. Kleihues P, Sobin LH: World Health Organization classification of tumors. Cancer 2000, 88(12):2887.

23. Lai JF, Xu WN, Noh SH, Lu WQ: Effect of World Health Organization (WHO) Histological Classification on Predicting Lymph Node Metastasis and Recurrence in Early Gastric Cancer. Medical science monitor : international medical journal of experimental and clinical research 2016, 22:3147-3153.

24. Nakamura K, Sugano H, Takagi K: Carcinoma of the stomach in incipient phase: its histogenesis and histological appearances. Gan 1968, 59(3):251-258.

25. Chai NL, Ling-Hu EQ, Morita Y, Obata D, Toyonaga T, Azuma T, Wu BY: Magnifying endoscopy in upper gastroenterology for assessing lesions before completing endoscopic removal. World J Gastroenterol 2012, 18(12):1295-1307.

26. Zhao Y, Feng F, Zhou YN: Stem cells in gastric cancer. World J Gastroenterol 2015, 21(1):112-123.

27. Kobayashi M, Takeuchi M, Ajioka Y, Hashimoto S, Sato A, Narisawa R, Aoyagi Y: Mucin phenotype and narrow-band imaging with magnifying endoscopy for differentiated-type mucosal gastric cancer. J Gastroenterol 2011, 46(9):1064-1070.

28. Chiu PWY, Uedo N, Singh R, Gotoda T, Ng EKW, Yao K, Ang TL, Ho SH, Kikuchi D, Yao F et al: An Asian consensus on standards of diagnostic upper endoscopy for neoplasia. Gut 2019, 68(2):186-197.

29. Kanesaka T, Uedo N, Yao K, Ezoe Y, Doyama H, Oda I, Kaneko K, Kawahara Y, Yokoi C, Sugiura Y et al: A significant feature of microvessels in magnifying narrow-band imaging for diagnosis of early gastric cancer. Endosc Int Open 2015, 3(6):E590-596.

\section{Additional File Legend}

Additional file 1: Table S1. The MS and MV patterns and histopathological types. (XLSX)

\section{Figures}




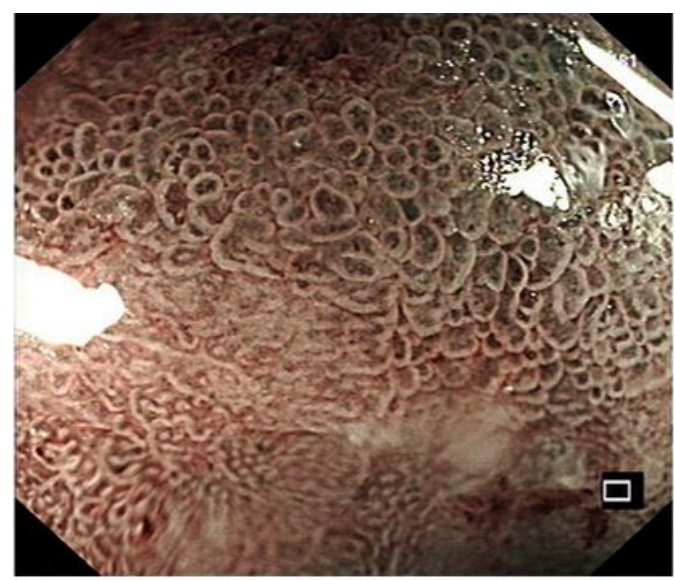

IV

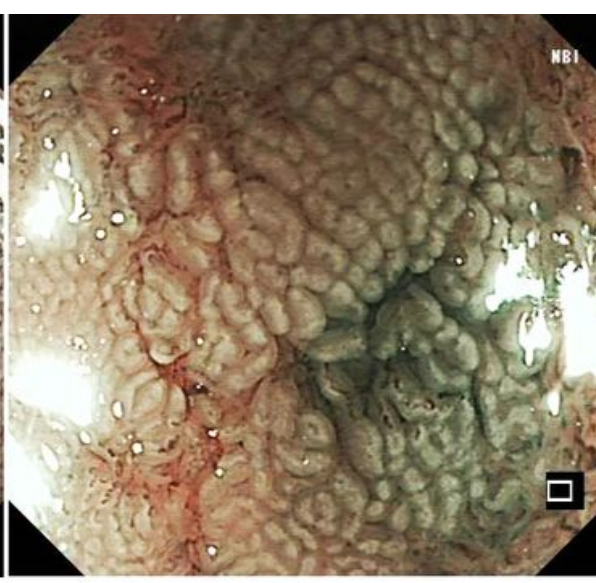

$\mathbf{V}_{1}$

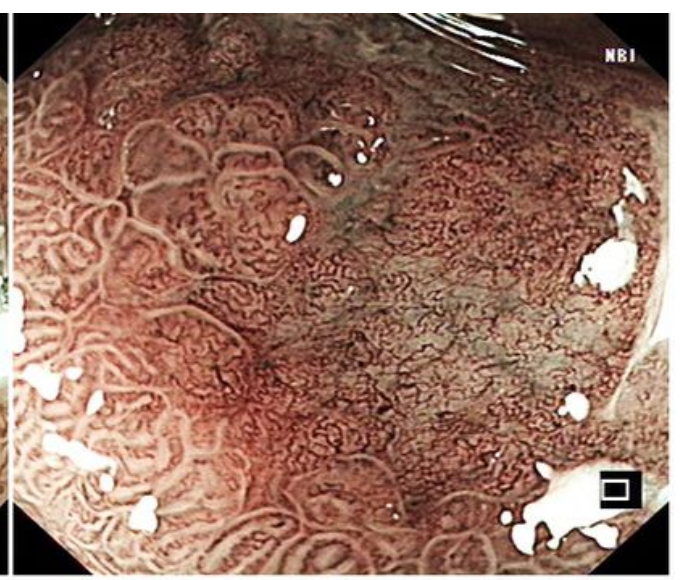

VI

\section{Figure 1}

Microsurface pattern in EGCs on ME-NBI.

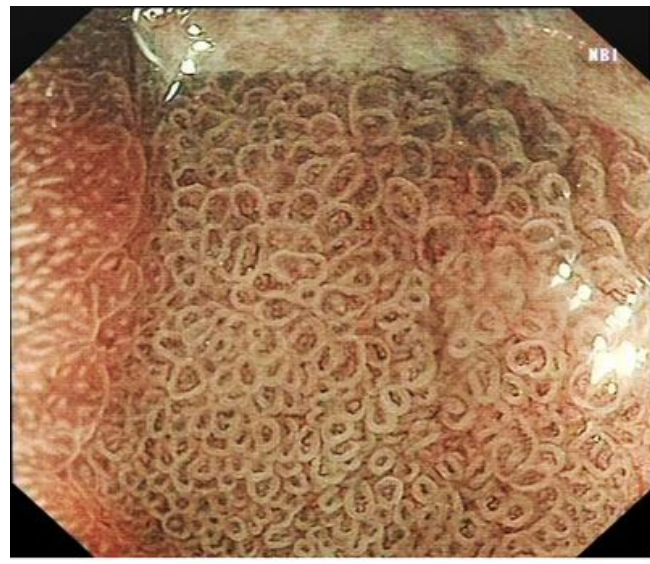

Loop

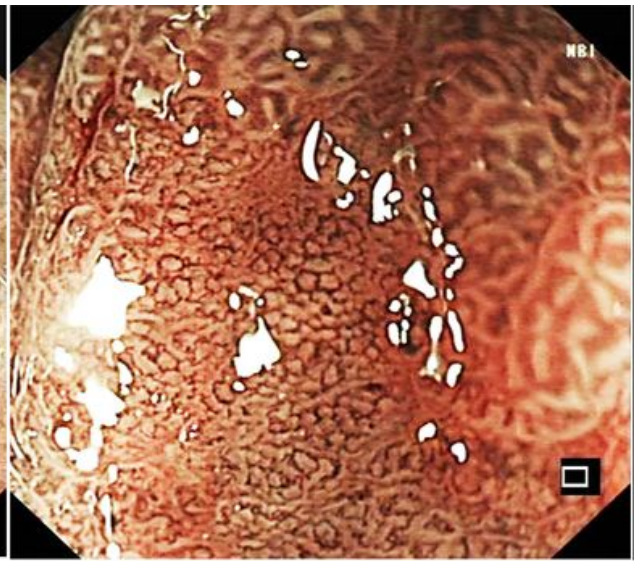

Fine network

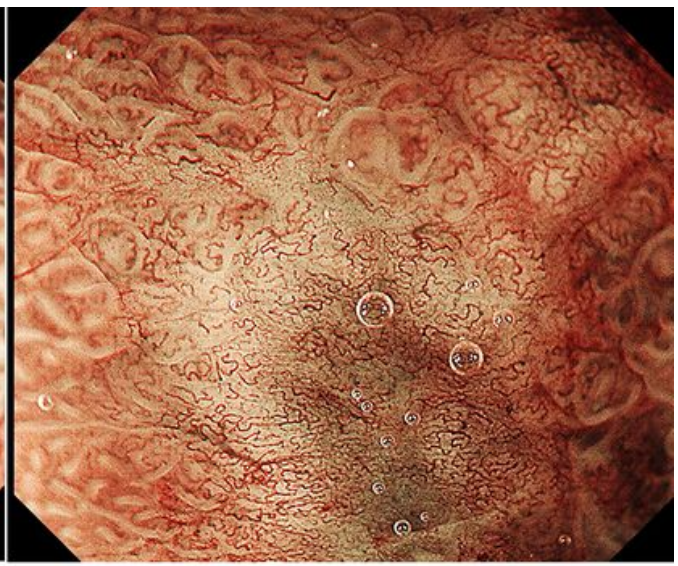

Corkscrew

Figure 2

Microvascular pattern in EGCs on ME-NBI. 


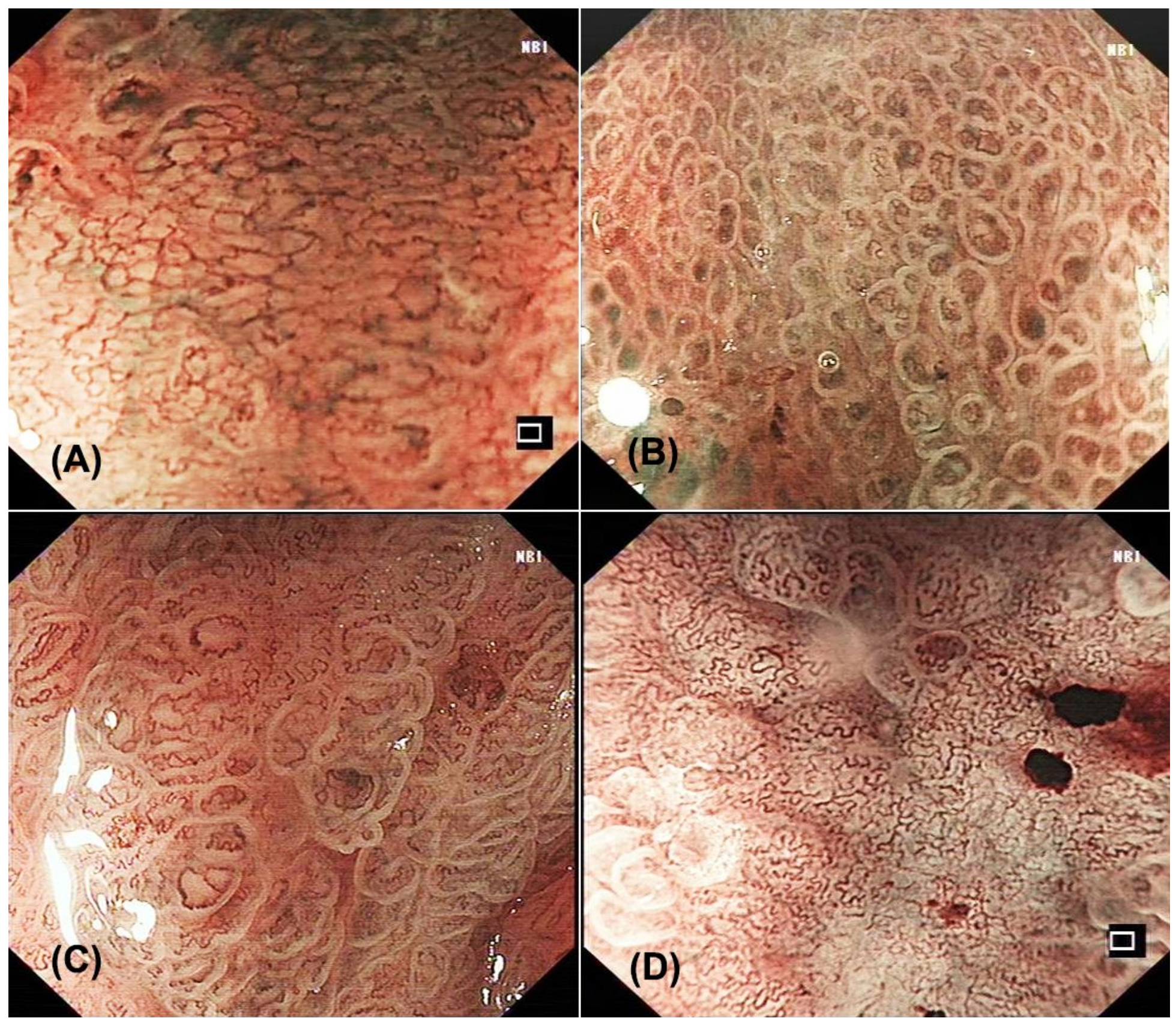

Figure 3

Narrow band imaging magnifying classification. (A) Fine network pattern (FNP); (B) Intralobular loop pattern 1 (ILL-1); (C) Intralobular loop pattern 2 (ILL-2); (D) Corkscrew pattern (CSP).

2

\section{Figure 4}

Comparison between the cases of high-grade intraepithelial neoplasia (HGIN) in corpus and antrum. (A) and (B) : A case of flat-type early gastric cancer shows $\mathrm{VI}$ type in the cardia. Histologic examination (C, HE staining) shows HGIN. (D) and (E): A case of depressed early gastric cancer shows IV type in the anterior wall of antrum. Histologic examination (F, HE staining) shows HGIN.

展 


\section{Figure 5}

Comparison between differentiated-type ECGs in corpus and antrum. (A) and (B) : A case of flat-type early gastric cancer near the cardia shows VI type. Histologic examination (C, HE staining) shows differentiated-type adenocarcinoma in the mucosa. (D) and (E) : A case of flat-type early gastric cancer in the antrum shows IV type. Histologic examination (F, HE staining) shows differentiated-type adenocarcinoma in the mucosa.

\section{Figure 6}

Comparison between undifferentiated-type ECGs in corpus and antrum. (A) and (B) : A case of depressed early gastric cancer shows VI type in the greater curvature of corpus. Histologic examination (C, HE staining) shows poorly differentiated tubular adenocarcinoma in the mucosa and parts of the lesion shows a signet ring cell carcinoma. (D) and (E): A case of depressed early gastric cancer shows IV type in the anterior wall of antrum. Histologic examination (F, HE staining) shows a signet ring cell carcinoma in the mucosa.

\section{Supplementary Files}

This is a list of supplementary files associated with this preprint. Click to download.

- Additionalfile1.xlsx 